\title{
Wasseranalyse nach RKI-Empfehlung
}

\author{
In der Zahnarztpraxis gehört die Behandlungseinheit zu den wichtigsten Medizinprodukten, mit \\ denen alle Patienten und auch das zahnmedizinische Personal in Kontakt kommen. Aus diesem \\ Grund muss stets eine hygienisch einwandfreie Funktionsweise gewährleistet sein, insbesondere \\ hinsichtlich des verwendeten und direkt mit dem Patienten in Kontakt kommenden Betriebswassers.
}

Für die Aufbereitung der Instrumente wird ein großer Aufwand betrieben, um zum Beispiel Übertragungsinstrumente nach validierten Verfahren zu reinigen, zu desinfizieren und zu sterilisieren. Diese Übertragungsinstrumente werden dann mit einer Behandlungseinheit verbunden, für deren Betrieb und Reinigung beziehungsweise Desinfektion solche strikten Vorgehensweisen nicht definiert sind. Damit besteht die Gefahr, dass das Instrument bei der Behandlung durch das Wasser der Behandlungseinheit kontaminiert wird. Dies stellt zum einen eine $\mathrm{Ge}$ fahr für Patienten und das behandelnde Personal dar und kann zum anderen auch die Lebensdauer der Übertragungsinstrumente negativ beeinflussen. Um die richtigen Maßnahmen für eine Dekontamination der Behandlungseinheit identifizieren zu können, ist eine regelmäßige mikrobiologische Untersuchung dringend erforderlich.

\section{RKI-Empfehlung: \\ Ermittlung der Gesamtkeimzahl pro 100 Milliliter}

Die Empfehlung zur Infektionsprävention in der Zahnheilkunde des Robert-Koch-Instituts (RKI) beschreibt mikrobiologische Untersuchungen des Betriebswassers, die helfen, die Einhaltung dieser Anforderungen sicherzustellen. Grundsätzlich soll die Gesamtkeimzahl pro 100 Milliliter ermittelt werden, um eine quantitative Aussage über die allgemeine Keimbelastung zu bekommen. Im Wasser, das mit dem Patienten in Kontakt kommt, ist darüber hinaus eine mögliche mikrobiologische Belastung mit Legionellen besonders kritisch. Legionellen kommen in natürlicher Form in Gewässern vor. Sie können aber vor allem durch Einatmen zur gefährlichen Legionärskrankheit führen.
Daher ist gerade auch beim zerstäubten Wasser an Behandlungseinheiten eine potenziell erhöhte Gefährdung des Patienten und der behandelnden Person gegeben. Bei Behandlung von immunsupprimierten Patienten ist zudem eine Untersuchung auf Pseudomonas aeruginosa sinnvoll. Die einzuhaltenden Grenzwerte bei Legionellen und Pseudomonaden sind in der RKI-Empfehlung beschrieben.

Werden Überschreitungen festgestellt, ist es erforderlich, mit dem Hersteller oder Installateur der Behandlungseinheiten über geeignete Desinfektionsmaßnahmen zu sprechen. Eine gängige Methode ist aktuell die Desinfektion mit Wasserstoffperoxid. Dieses Verfahren kann aufgrund der korrodierenden Eigenschaften auch Nachteile mit sich bringen. In jedem Fall sollte hier nach einem umfassenden Konzept gesucht werden, das sowohl die Besonderheiten der verwendeten Behandlungseinheit als auch der eingesetzten Instrumente berücksichtigt.

Die Wasseranalyse an Dentaleinheiten erfolgt nicht nach der Trinkwasserverordnung, sondern auf Grundlage der RKI-Empfehlung. Das impliziert unter anderem, dass die Probenahme durch einen geschulten Praxismitarbeiter erfolgen kann und dass keine Meldepflicht besteht, wenn zum Beispiel eine erhöhte Belastung durch Legionellen festgestellt wird. Die Behörden fordern die Analyse nach RKI-Empfehlung im jährlichen Turnus als einen zusätzlichen Bestandteil des Hygiene-Managements.

ValiTech kann bei der Wasseranalyse nach der RKI-Empfehlung unterstützen und bietet eine einfach vor Ort durchzuführende Probenahme und die Laboranalyse inklusive Zertifikat nach akkreditierten Verfahren.

Alexander Huhn, ValiTech GmbH \& Co.KG

Durch eine Rahmenvereinbarung mit dem Freien Verband Deutscher Zahnärzte (FVDZ) bietet die Firma ValiTech eine günstige Möglichkeit zur mikrobiologischen Untersuchung des Betriebswassers von Dentaleinheiten nach der RKI-Empfehlung „Infektionsprävention in der Zahnheilkunde". Dabei werden FVDZ-Mitgliedern vergünstigte Konditionen geboten. Der Praxis wird ein Prüfpaket zur Probenahme vor Ort zugeschickt, an schließend folgen die Auswertung im Labor und die Erstellung eines Prüfberichts. Nähere Informationen zur Kontaktaufnahme und zu Leistungen von ValiTech unter www.valitech.de.
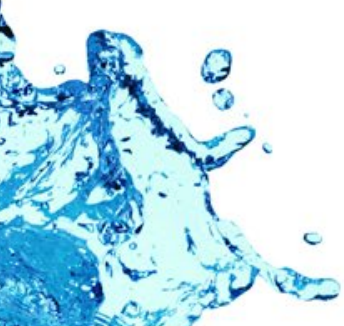